\title{
Zigbee wireless sensor network localization evaluation scheme with weighted centroid method
}

\author{
Lonesy Thammavong ${ }^{1}$, Khamphong Khongsomboon ${ }^{1}$, Thanadol Tiengthong ${ }^{2}$ and Sathaporn Promwong ${ }^{2, *}$ \\ ${ }^{1}$ Department of Electronics and Telecommunication Engineering, Faculty of Engineering, National University of Laos Vientiane, LAOS \\ ${ }^{2}$ Department of Telecommunications Engineering, Faculty of Engineering, King Mongkut's Institute of Technology Ladkrabang, \\ Bangkok, Thailand
}

\begin{abstract}
Using wireless communication system, appropriate and correct indoor localization with Zigbee sensor network and could provide interesting services and applications. In this study the Zigbee transmission model with positioning method by using the relative-span exponentially weighted centroid method for the indoor localization. The experimental results and analyze results are evaluated a distance error. The ZigBee transmission model in measurement consists of 121 positions with distance between positions to positions is 0.3 meter. The experimental setup at every position operated at frequency band from $2.3 \mathrm{GHz}$ to $2.5 \mathrm{GHz}$. The accuracy of estimated position is considered in the term of distance error with the cumulative distribution function (CDF) of distance error is shown. The result presents optimal value for REWL is 0.2 and mean of distance error is $0.65 \mathrm{~m}$.
\end{abstract}

\section{INTRODUCTION}

The Zigbee wireless networks are widely used in several applications for indoor and outdoor localizations. The information of localization is a main criterion for the capability of wireless sensor network. The wireless network cannot work properly without position information. Moreover, the accurate location estimation is helpful in wireless sensor networks (WSNs) services such as routing, information processing [2], [3], and [5]. Thus, in WSNs, localization has become a very popular research orientation. Based on the requirement for measuring the received signal strength indicator (RSSI) between reference nodes and target nodes in the positioning process, WSNs localization algorithm can be split into the range-based localization algorithm and the range-free localization algorithm. A good localization, algorithm should be immune to ambient environmental impacts as well as inexplicit distances and should calculate a position as soon as possible. A promising algorithm of localization is the REWL algorithm with Zigbee technology.

The Zigbee is short-range wireless technology, lowcost and low power which satisfies IEEE 802.15.4 standard for both physical layer (PHY) and medium access control (MAC) [8]. The advantage of the Zigbee are time-saving and high reliability, distance ranging from 10 meter to 100 meter, suitable for construction, industrial control, work environment, Data rate ranging from $125 \mathrm{kbps}$ to $250 \mathrm{kbps}$ and medical

There are many techniques with positioning method. It's available either based on the hardware arrangement, or relied on simulation algorithm. In this research, the performance of WSNs depends on the density of nodes and the quality of data signals. The precision of position could be calculated from the co-ordinations of any four reference nodes.

In this paper we consider algorithm for indoor localization resolution. We evaluation the Weighted Centroid method based on RSSI for WSNs. The estimation of position is considered in term of distance error. The following part of this paper is arrange as follows. Section II explain of evaluation of indoor localization. Next, the measurement setup and results of this paper are explained in Section III and IV. Eventually, summary the conclusions are given in section $\mathrm{V}$.

\section{EVAULTION METHOD OF INDOOR LOCALIZATION}

\subsection{Receive Signal Strength Indicator (RSSI)}

The RSSI (Receive Signal Strength Indicator) is a method to measure the strength of radio waves becomes different depending on the space between a reference node and a target node. The strength of received signals can compare by using probability distribution based on a statistical method and measures the location. To use this method, it should measure strengths of sample signals at predefined various locations in advance. The Zigbee used in sensor networks provides a REWL algorithm with RSSIs.

\footnotetext{
* Corresponding author: sathaporn.pr@kmitl.ac.th
} 


\subsection{Relative-Span Exponentially Weighted Localization Algorithm (REWL)}

The REWL algorithm is based on Relative Weighted Localization (RWL) for wireless sensor networks proposed in [1] which the REWL is better than RWL. We show difference between two follow equations 1 and 2. This solution show how to approach the location $p$ of the target node by calculating the RWL of the coordinate. More precisely, the position of the estimated target node is given by.

$$
p=\frac{\sum_{i=1}^{n}\left[R S S_{\max }-R S S_{i} \times a_{i}\right]}{\sum_{i=1}^{n}\left[R S S_{\max }-R S S_{i}\right]}
$$

While RWL performs solely average of the coordinates of reference node devices to localize blind folded devices, the REWL uses weights to guarantee an improvement of localization. establishing from the calculation of the RWL equation 1, the equation for determining the position with REWL is derived. Reveal the term as sum of ones and the multiplication of with ones, equation 1 is expanded to the REWL equation 2.

The REWL is an RSSI-based range-free localization algorithm. This algorithm is encouraged by the WCL method [4], [6], [7], [8] The weights are acquired by the respective positioning of the anchor RSSI value inside the span of all the RSS values measured by the target node. In the estimation of the target position, the REWL algorithm oblige the reference nodes, which present higher RSSI values and hence are probable to be closer to the target node. This is earning by used the weighting factor $\lambda$, as state by the exponentially moving average concept. The estimated target node position is given by

$$
p=\frac{\sum_{i=1}^{n}\left[(1-\lambda) R S S_{\max }-R S S_{i} \times a_{i}\right]}{\sum_{i=1}^{n}\left[(1-\lambda) R S S_{\max }-R S S_{i}\right]}
$$

Where $n$ is number of reference node in range, $p$ is the REWL approximated position of target node, $R S S_{\max }$ is the highest value in the span of the RSSI values measured by the target nodes, $R S S_{i}$ is the RSSI value of the target node.

From equation 2 can be solution the equations 3 and 4

$$
\begin{gathered}
x=\frac{\left[(1-\lambda)^{R S S_{\max }-R S S_{i}} \times x_{1}\right]}{\left[(1-\lambda)^{\left.R S S_{\max }-R S S_{i}\right]}+\frac{\left[(1-\lambda)^{R S S_{\max }-R S S_{i}} \times x_{2}\right]}{\left[(1-\lambda)^{\left.R S S_{\max }-R S S_{i}\right]}\right.}\right.} \\
+\frac{\left[(1-\lambda)^{R S S_{\max }-R S S_{i}} \times x_{3}\right]}{\left[(1-\lambda)^{R S S_{\max }-R S S_{i}}\right]}+\frac{\left[(1-\lambda)^{R S S_{\max }-R S S_{i}} \times x_{4}\right]}{\left[(1-\lambda)^{R S S_{\max }-R S S_{i}}\right]}
\end{gathered}
$$

and

$$
\begin{aligned}
y & =\frac{\left[(1-\lambda)^{R S S_{\max }-R S S_{i}} \times y_{1}\right]}{\left[(1-\lambda)^{R S S_{\max }-R S S_{i}}\right]}+\frac{\left[(1-\lambda)^{R S S_{\max }-R S S_{i}} \times y_{2}\right]}{\left[(1-\lambda)^{R S S_{\max }-R S S_{i}}\right]} \\
& +\frac{\left[(1-\lambda)^{R S S_{\max }-R S S_{i}} \times y_{3}\right]}{\left[(1-\lambda)^{R S S_{\max }-R S S_{i}}\right]}+\frac{\left[(1-\lambda)^{R S S_{\max }-R S S_{i}} \times y_{4}\right]}{\left[(1-\lambda)^{R S S_{\max }-R S S_{i}}\right]}
\end{aligned}
$$

\subsection{Performance Evaluation}

The localization error is explain as a distance between the exact position and the approximated location of a target node.

\section{ZIGBEE WIRELESS SENSOR NETWORK MEASUREMENT}

In this research we have designed a model to measurement for location identifier of the target node in indoor environment. For more accurate localization, the experiment will be done without obstructions. RSSI measurement is carry on by using Zigbee in the term of transmission of the signal strength at frequency of 2.4 GHz. The model used for measurement consists of 121 positions with space between one position and next position is 0.3 meter. The model of measurement is depicted in figure 1 . For $\mathrm{x}$ and $\mathrm{y}$ axes on the coordinates $(0, \mathrm{~A}),(11, \mathrm{~A}),(0, \mathrm{~K})$ and $(11, \mathrm{~K})$ are used as reference nodes. The measurement was done at the room 305 of 3th floor, E-Building, Faculty of Engineering, King Mongkut's Institute of Technology Ladkrabang.

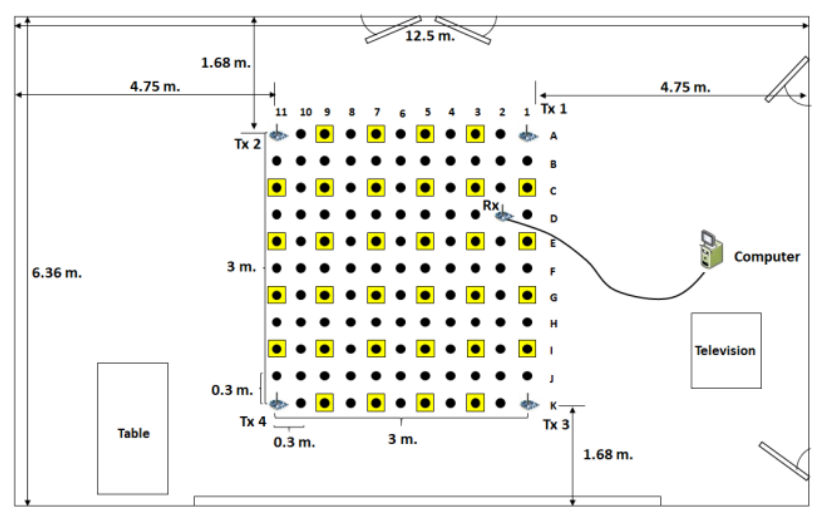

Fig. 1. The experimental setup.

\section{EXPERIMENTAL RESULTS}

In this section we proposed algorithm of performance analysis. By consider simulate the REWL based on WSNs for RSS. The accuracy calculates from reference node to target node follow equation 2 and the localization error calculates follow equation 5 . 


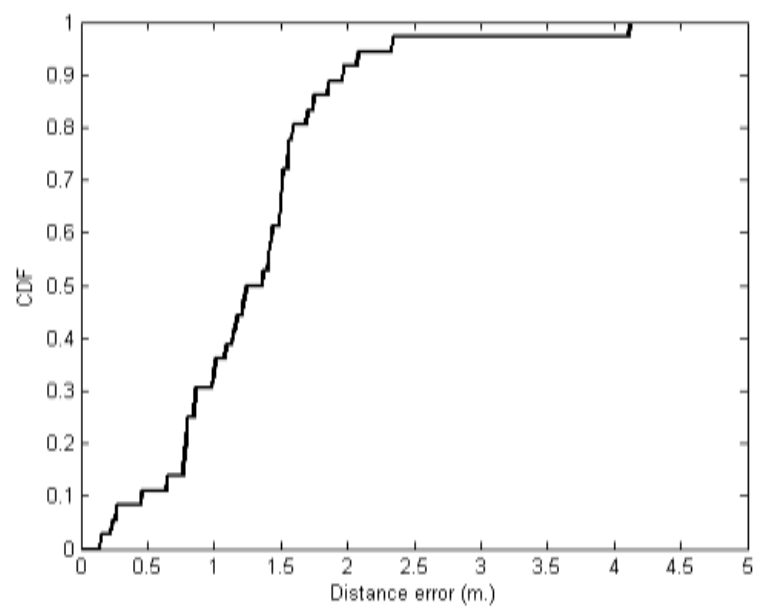

Fig. 2. The CDF of the distance error with range with different weighting factor at $\lambda=0.10$.

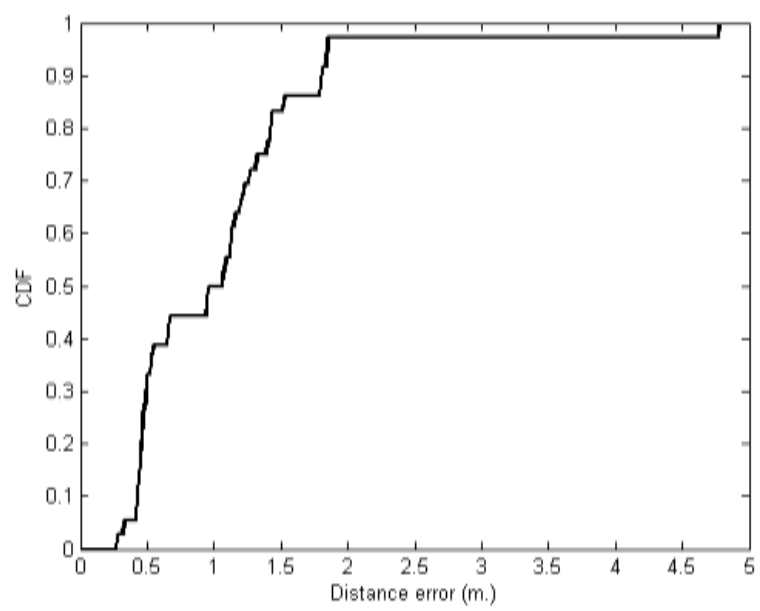

Fig. 3. The CDF of the distance error with range with different weighting factor at $\lambda=0.15$.

The distance error of each estimated distance is shown in term of CDF. The distance error in $\mathrm{x}, \mathrm{y}$ axes of REWL algorithm with $\lambda=0.1$ is shown in figure 2 the mean of distance error is $1.24 \mathrm{~m}$. The distance error in $\mathrm{x}$, $y$ axes of REWL algorithm with $\lambda=0.15$ is shown in figure 3 the mean of distance error is $0.9 \mathrm{~m}$. The distance error in $\mathrm{x}, \mathrm{y}$ axes of REWL algorithm with $\lambda=0.2$ is shown in figure 4 the mean of distance error is $0.65 \mathrm{~m}$. The distance error in $\mathrm{x}$, $\mathrm{y}$ axes of REWL algorithm with $\lambda=0.25$ is shown in figure 5 the mean of distance error is $0.75 \mathrm{~m}$. To ascertain the optimal $\lambda$, we simulated a full equipped sensor network of the enclosed $2 \times 2$ reference node (4 nodes). In the figures $2,3,4$, and 5 are demonstrating the CDF of the distance error with different weight factor $(\lambda)$. The simulation result shows several minimum distance errors depending on the distance and the weight factor $(\lambda)$. The smallest average of distance error exists at $\lambda=0.20$, yields the best result as shown in figure 4. Consequently, before begin the localization process, a concentrated analysis of the transmission range, the dimensions of the network and distance are required to get the smallest distance error.

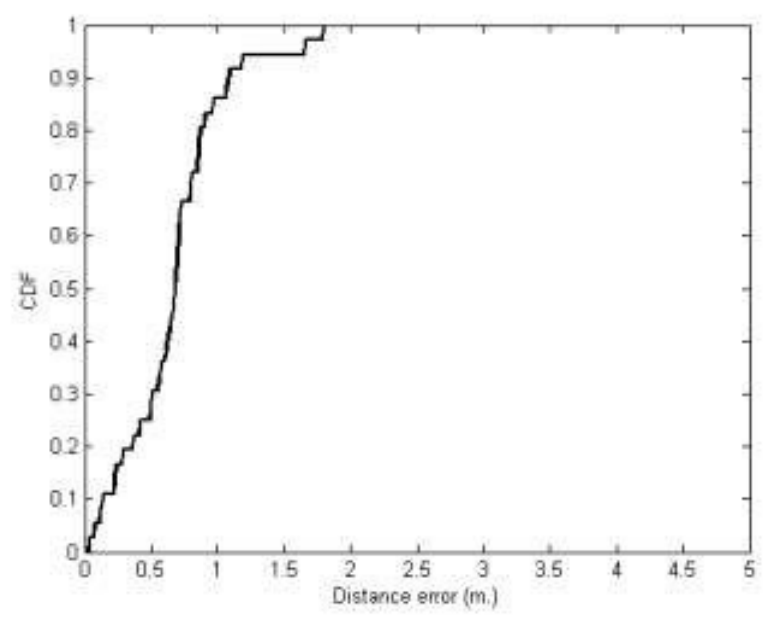

Fig. 4. The CDF of the distance error with range with different weighting factor at $\lambda=0.20$.

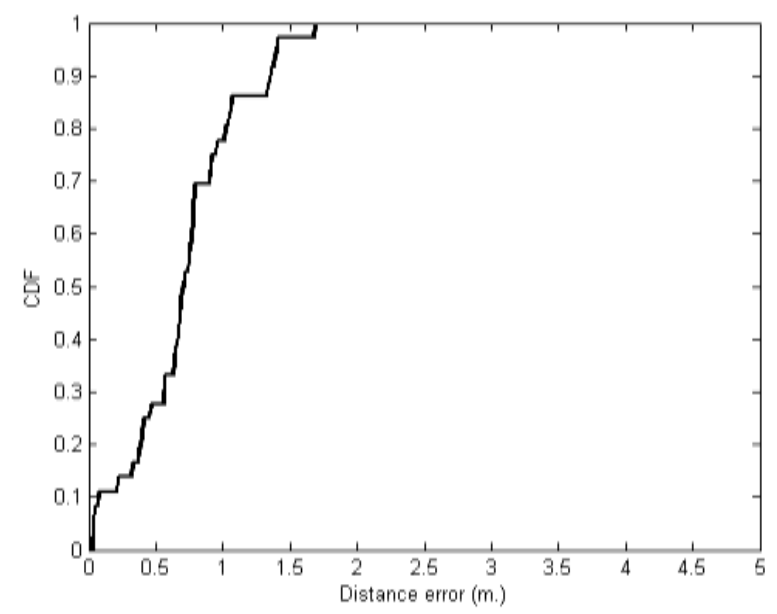

Fig. 5. The CDF of the distance error with range with different weighting factor at $\lambda=0.25$.

But in practice, interferences and obstacles as well as uncertain electronic transfer elements within the transceivers. Afterward, distances are estimated by using REWL algorithm. Consequently, the distance error of each position is evaluated by mesh contour and shown in figures $6,7,8$ and 9 .

\section{CONCLUSION}

This paper how to evaluate the Zigbee transmission model for indoor localization based on RSSI measurements collected by using REWL algorithm for WSNs. It provides the approximated position error of the target node in dissimilar test a points inside an inspection power transmission loss. This research we are summarizing the basic theoretical and practical facts impact the measured data exceedingly. Therefore, calculating distance using simple model will result in faulty distance with at least offset errors and scaling errors for concerning analysis of the distance error of REWL. 


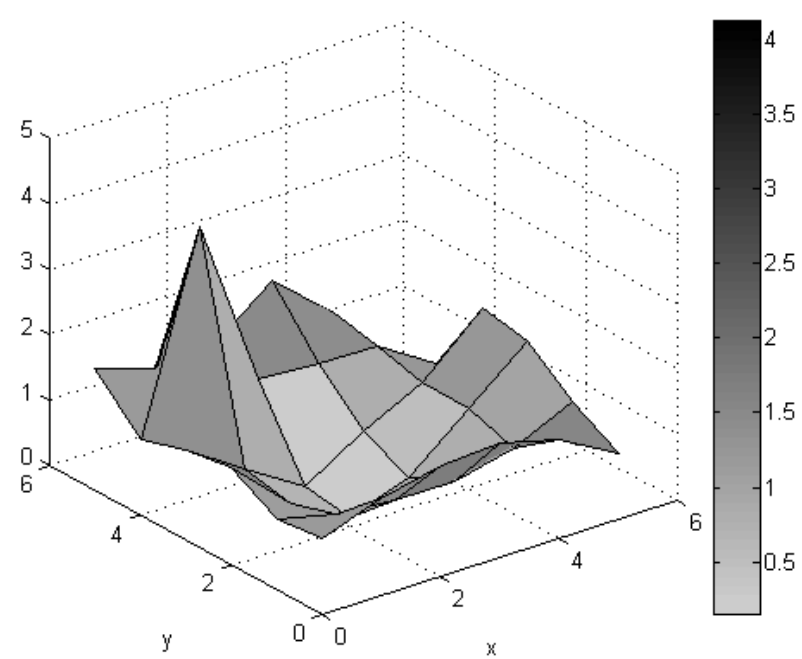

Fig. 6. The distance error of each position at $\lambda=0.10$.

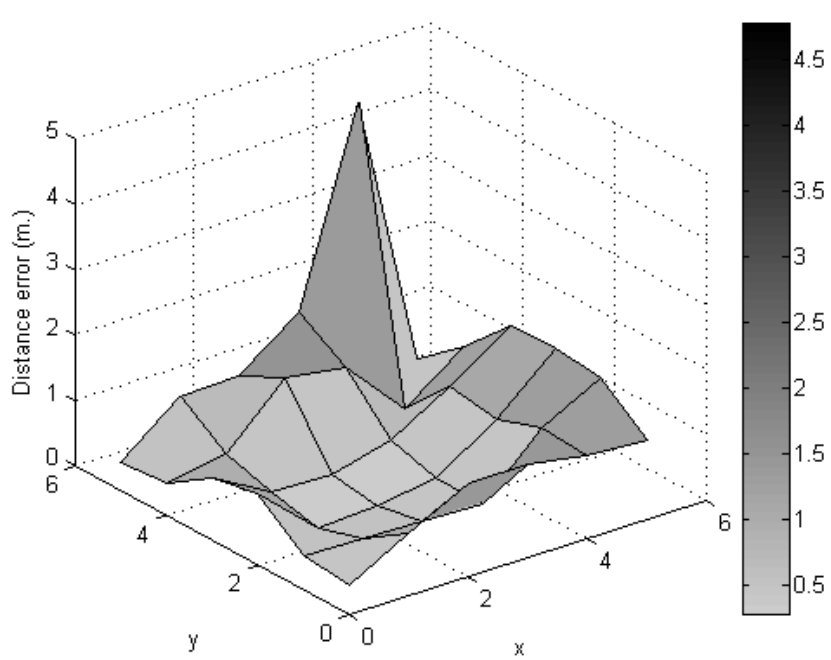

Fig. 7. The distance error of each position at $\lambda=0.15$.

Moreover, the REWL algorithm and against scaling error of distance measurement has been clarify. Localization results using Zigbee node indoor environment are described. Even though the positioning algorithm does not yet give the suitable results very precisely, the presented localization algorithm in merging with the Zigbee technology offer lots of advantages. It's very significant advantages are its minimum resource requirements, fast computation process and low complexity which advocate REWL as localization algorithm in Zigbee WSNs.

\section{References}

1. C. Laurendeau and M. Barbeau, "Relative span weighted localization ofuncooperative nodes in wireless networks," inProc. Int. Conf. WASA, pp. 358-367, August, 2009

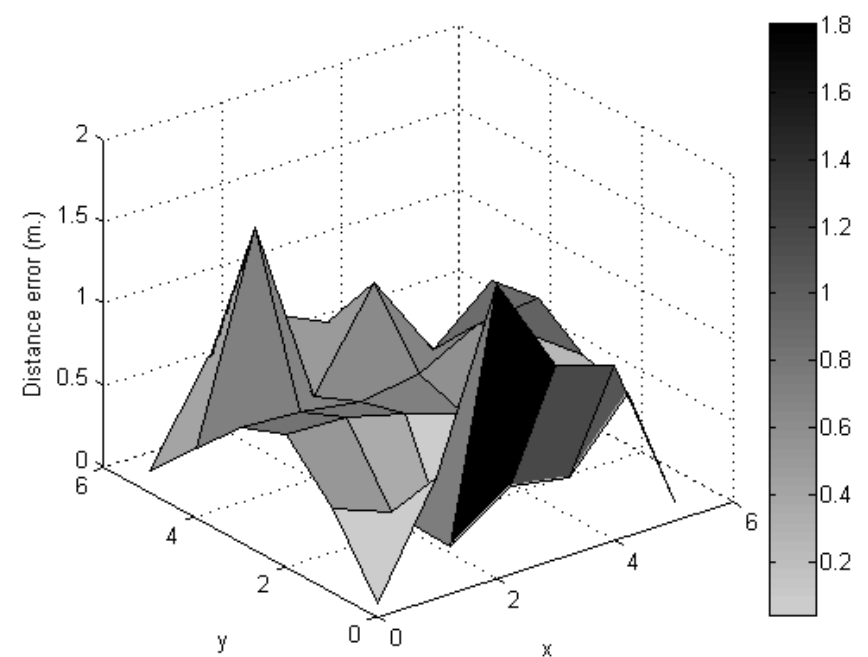

Fig. 8. The distance error of each position at $\lambda=0.20$.

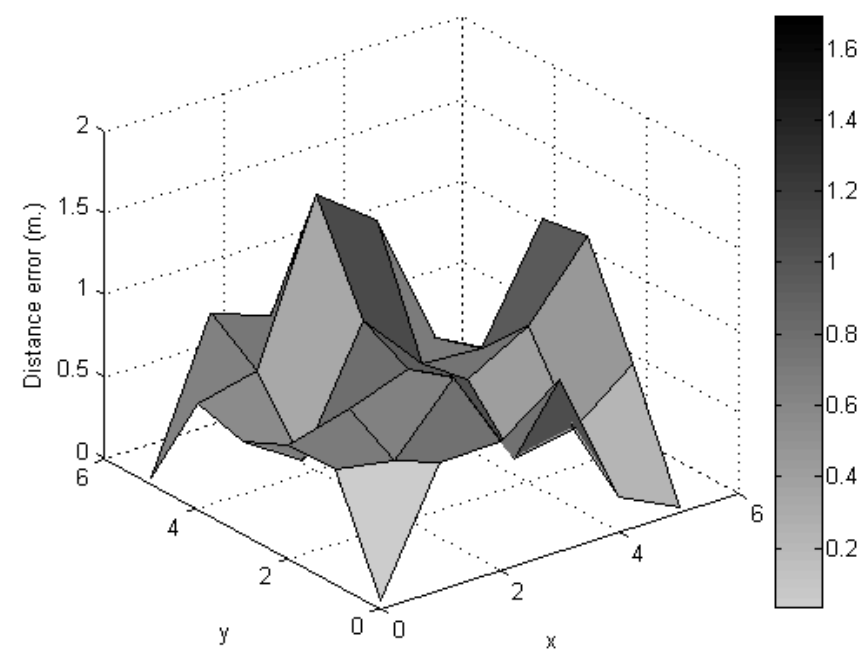

Fig. 9. The distance error of each position at $\lambda=0.25$.

2. F lan. Akyildiz, W. Su, Y. Sankarasubramaniam, and E. Cayirci, "A survey on sensor network", IEEE Communication Magazine, pp102- 114, August 2002

3. H. Liu, H. Darabi, P. Banerjee, and J. Liu, "Survey of wireless indoor positioning techniques and systems," Systems, Man, and Cybernetics, Part C: Applications and Reviews, IEEE Transactions on, vol. 37, no. 6, pp. 1067-1080, November, 2007

4. J. Blumenthal, R. Grossmann, F. Golatowski, and D. Timmermann, "Weighted centroid localization in Zigbee-based sensor networks," in Proceedings of the IEEE International Symposium on Intelligent Signal Processing (WISP '07), Madrid, Spain, pp.16, October, 2007.

5. J. Blumenthal, R. Grossmann, F. Golatowski, and D. Timmermann, "Weighted centroid localization in Zigbee-based sensor networks," inProceedings of the IEEE International Symposium on Intelligent Signal Processing (WISP '07), Madrid, Spain, pp.16, October, 2007 
6. J. Blumenthal, F. Reichenbach and D. Timmermann: Position Estimation in Ad hoc Wireless Sensor Networks with Low Complexity. In Joint 2 nd Workshop on Positioning, Navigation and Communication and 1st Ultra-Wideband Expert Talk, pp. 41-49, March, 2005.

7. Kristalina, P., Wirawan \& Hendrantoro, G., "Improved Range-free Localization Methods for Wireless Sensor Networks", Proc. 2011 Int'l Conf. on Electrical Engineering and Informatics (ICEEI), pp. I5-1, July, 2011.

8. S. Schwarzer, M. Vossiek, M Pichler, A. Seltzer, "Precise Distance Measurement with IEEE 802.15.4 (Zigbee) Devices", Radio and Wireless Symposium IEEE, pp.779-782, January, 2008.

9. Y Chen, Q Pan, Y Liag, Z Hu, “Adaptive Weighted Centroid Target Localization Algorithm Based on RSSI in WSN", Computer Science and Information Technology (ICCSIT), 3rd IEEE International Conference, pp. 331-336, July, 2010. 